Revista

\title{
Multi-Ensayos
}

Vol. 7, núm. 14

ISSN: 2412-3285

https://multiensayos.unan.edu.ni

DOI: https://doi.org/10.5377/multiensayos.v7i14.12003

\section{Formación docente constante para una comunicación virtual efectiva}

\section{Continuous teacher training for effective virtual communication}

\author{
María José Muñoz González
}

Recibido: 06 de mayo de 2021. Aceptado: 14 de junio de 2021

\section{RESUMEN}

El presente ensayo se basa en el supuesto que "La Formación Docente debe ser constante y efectiva en la Comunicación Virtual". En la actualidad los profesionales de la educación deben manejar una comunicación efectiva, pues la enseñanza expuesta a constantes cambios debe garantizar la enseñanza y aprendizaje haciendo uso de las tecnologías, al mismo tiempo que el profesorado debe actualizar sus conocimientos de forma dinámica y en constante cambio. Los docentes hoy son protagonistas de un cambio profundo en las metodologías educativas y para ello requieren de capacitaciones progresivas, las plataformas online educativas tecnológicas nos invitan a la innovación pedagógica y acercamiento a las nuevas generaciones de estudiantes, en consecuencia, las clases presenciales deben ser transformadas a la virtualidad con cambios profundos de formatos educativos, para que el educando participe de su aprendizaje Virtual.

Palabras claves: Comunicación; profesores; formación; educación; tecnologías.

\section{ABSTRACT}

This essay is based on the assumption that "Teacher training in the realm of Virtual Communication has to be continuous and effective". Nowadays, education professionals have to be proficient in effective communication, given that the field of education is open to constant changes that requires adapting to new technologies in order to guarantee teaching and learning outcomes. In this context, practitioners need to update their knowledge-base in a continuously dynamic manner. Teachers are hence the main protagonists of deep transformations in teaching methodologies, for which they require progressive training - in particular for implementing distance-learning spaces through technological platforms. These kinds of virtual spaces provide a venue for teaching innovations as well as for enhanced involvement of new generations of students. This entails transforming face-to-face classes into virtual formats by operating deep changes in teaching approaches, in order to allow students to take an active part in virtual learning formats.

Keywords: Communication; teachers; training; education; technologies.

1 Estudiante Programa de Doctorado en Educación. Universidad Bernardo O’Higgins. UBO (Chile). Correo electrónico: mariamu@ postgrado.ubo.cl. Orcid: https://orcid.org/0000-0002-3495-7607

(c) 2021 Revista Multi-Ensayos.

(c) (i) Este trabajo está licenciado bajo una Licencia Internacional Creative Commons 4.0 Atribución-NoComercial-Compartirlgual. 


\section{INTRODUCCIÓN}

La docencia es la esencia de la enseñanza, por este motivo es preciso reconocer el trabajo de los profesores, la importancia de la comunicación efectiva y las competencias Tecnologías de Información y Comunicación conocidas como TICs que deben estar presentes desde la formación inicial, la formación a través del tiempo y el ejercicio de la docencia. En la actualidad se observan cambios importantes, adaptaciones curriculares, metodológicas junto a un gran cambio gatillado por la comunicación online al interior de la comunidad escolar y en todo ámbito educativo incluyendo la universitaria. Villarreal-Villa et.al, (2019) nos indica que, las nuevas tecnologías de la información han sido elementos influyentes para la transformación en diversos ámbitos de nuestras vidas.

La educación ha cambiado y la pandemia Covid19 aceleró el avance del uso de aparatos electrónicos por ejemplo celulares, computadores. laptop, tablets, entre otros, para generar comunicación entre estudiantes, profesores, apoderados, compañeros de trabajos y clases virtuales, y brindar información destinada al aprendizaje, por consiguiente, los profesores han debido reaprender su trabajo. A este respecto, los profesores chilenos son reconocidos a nivel internacional por su excesivo trabajo docente, según estudio de la OECD (2020), TALIS 2018 la cantidad de horas anuales son 2.006, de las cuales 1.146 son horas de clases y en algunos casos, la cantidad de estudiantes por aula en algunos casos superan los 40 educandos generando sobre trabajo, los profesores han ofrecido sus aplicaciones personales como WhatsApp, Facebook, Instagram, Correos electrónicos no institucionales, para el uso educativo, traspasando los límites establecidos hacia los alumnos y apoderados.

Los profesionales de la educación a pesar de estar formados académicamente, éstos deben seguir constantemente capacitándose, para lograr la actualización de conocimientos, para ejercer las clases online. Los postítulos, menciones o posgrados deben ser pagados por ellos, para estar al nivel académico requerido por la alta exigencia laboral a la cual deben responder. Los docentes requieren de competencias actualizadas enfocadas en la Educación Virtual. A este respecto, Villarreal-Villa et.al, (2019) plantean que existe un cambio tecnológico constante donde los estudiantes de pedagogía y profesores en ejercicio necesitan constante capacitación y reintegro de saberes.

\section{Educación Virtual, nuevas habilidades y la importancia de las instituciones educativas}

Los escenarios actuales de la educación apuntan al uso de aplicaciones como WhatsApp, Telegram, correos electrónicos no formales, llamados telefónicos de forma simétrica entre pares y asimétricas entre profesores y jefaturas, profesores estudiantes, todo lo cual ha generado la demanda de un exceso de trabajo desarrollando agobio y estrés laboral. La educación requiere no solo de profesores titulados y profesionales competentes en el área educacional, se observa que esta realidad no solo cruza fronteras nacionales e internacionales, sino también la intimidad de los hogares y espacios familiares. Para dicha demanda se necesitan nuevas competencias docentes, y nuevas habilidades para cambiar de paradigma educativo. Villarreal-Villa et.al, (2019) indican que la incorporación de herramientas y recursos contemporáneos enfocados en el aprendizaje de los educandos, donde los profesores sean 
los protagonistas de la innovación pedagógica, se debe incorporar la cultura digital a través de un comportamiento ético y legal en las prácticas profesionales.

Consecuente con lo anterior, la pandemia del coronavirus conocida COVID19 ha visibilizado una brecha de diferencias sociales $y$, debido a que los docentes deben comunicarse por vías digitales con sus estudiantes, ello ha significado un proceso difícil de afrontar por las instituciones educativas. En efecto, existen alumnos que no disponen de aparatos tecnológicos adecuados para recibir la enseñanza virtual específicamente mediante aulas en línea, videoconferencias, videos, programas educativos virtuales. De igual modo no existe una política pública que considere la capacitación nacional de profesores mediante una plataforma única para la realización de clases online y offline. A ello se suma la necesidad imperiosa de continuar asegurando la calidad en la educación en Chile como también ocurre en otros países de la región. Tal como plantea Hurtado Talavera (2020) la realidad mundial ha cambiado con el COVID19 y la formación docente y la comunicación efectiva han modificado la realidad mundial. Hoy día estamos enfrentados a nuevos cambios educativos, familiares y simbólicos; también la metodología educativa se ha modificado y es necesario que los profesores se adapten a esta nueva realidad para generar efectividad en la comunicación con la finalidad de mejorar la educación.

Si bien es cierto pensar en un regreso a clases tal como se conocía, antes de la pandemia, es ilusorio por cuanto el futuro educativo no continuará como era antes de dicha crisis sanitaria. En este sentido cuidar la salud de estudiantes, profesores, asistentes de la educación, familias, de todos los actores de la comunidad escolar, es tan importante como aprender, pues es parte del aprender a vivir juntos para salvaguardar la salud pública. Sin duda ello es parte de la nueva realidad mundial donde debemos pensar en la salud del otro, donde los docentes deben crear nuevas estrategias, metodologías de aprendizaje quizás aún no conocidas las cuales deben ser creadas. Instan a que la educación vaya en la misma dirección de los procesos de transformación cultural y social generados alrededor de las TICs, donde la pandemia nos guía por un nuevo camino lleno de cambios radicales en el ámbito educativo.

Por otra parte, entre los elementos relevantes a considerar se encuentra la formación docente enfrentando un macro cambio de paradigmas. Esta realidad, según Suárez Huz et.al, (2018) se encuentra en constante evolución y crecimiento, y combina la presencia de las TICs, modelos culturales y formas de enseñanza y aprendizajes. En otras palabras, hoy se puede trabajar a distancia gracias al desarrollo de las tecnologías, aunque ello es posible sólo para un porcentaje de la población que tiene los medios y recursos necesarios para estudiar de forma remota. La educación se encuentra en una fase de reacomodación en la que destaca la internalización, que incorpora las tecnologías, los medios de expansión, la era de la información, y la inmediatez. Reimers, et.al, (2020) plantean que las diferencias entre sistemas escolares en su capacidad para diseñar e implementar respuestas educativas efectivas durante la contingencia, amplificarán las brechas de oportunidades en todas las jurisdicciones, esto afectaría las brechas educativas. 


\section{Comunicación por medio de la tecnología}

En consonancia con lo explicado anteriormente se hace imperativo que dicha comunicación carente de garantías sociales y falta de recursos tecnológicos, entre otros, el actual problema sea afrontado por los distintos países de la región latinoamericana para no perjudicar especialmente a la población más desfavorecida, por cuanto muchos educandos en el último tiempo no han logrado acceder a la educación virtual lo que es fundamental para el aprendizaje y el desarrollo de la educación en el Continente Americano. El profesor debe manejar medios tecnológicos para realizar sus clases, han debido aprender de ellos en corto tiempo, para ofrecer una formación adecuada para entregarla a sus estudiantes y, al mismo tiempo, mejorar sus propias prácticas. Suárez Huz et.al, (2018) explican y enfatizan la atención a elementos culturales, sociales, afectivos y cognitivos que intervienen en el momento de emplear las TICs en la educación. Cuando se inventó la rueda en el tiempo de los Sumerios (3.000-2.350 a.C.) considerando que fueron la primera civilización urbana, difícilmente se siguió ocupando la palanca para el movimiento de objetos pesados, de manera similar la educación debe avanzar al ritmo de las tecnologías, y los docentes deben ser la rueda que empuja este gran carro del aprendizaje, y no convertirse en un obstáculo.

En efecto, la realidad que hoy conocemos con la comunicación virtual, Ilamados telefónicos, mensajería instantánea, entre otras, van a quedar instauradas en el colectivo educacional pues, si bien es cierto se ha avanzado tecnológicamente en la educación, hoy es posible una comunicación efectiva sin estar en el mismo lugar físico; al mismo tiempo la tecnología permite romper fronteras en los horarios como en las relaciones interpersonales. Almazán Gómez. et.al, (2020) plantean la posibilidad de que esta experimentación masiva suponga una aceleración de un proceso de digitalización, y la comunicación debe ser un destino inevitable. Asumiendo que su incorporación no es opcional entonces los docentes deben conocer y manejar la comunicación virtual por medio de la tecnología.

La responsabilidad del profesor (a) es generar nuevos métodos de innovación pedagógica, por ejemplo, el modo híbrido de aprendizaje que plantea la entrega de conocimientos e interacción donde el estudiante debe estar atento a las indicaciones del profesor que es el conocedor del contenido el cual puede ser entregado de diversas formas sincrónica y asincrónica mediante la clase virtual. En la literatura especializada el estudio realizado por Munro. et.al, (2018) se releva que la mercantilización aumenta las desigualdades sociales, la educación y la formación docente, la responsabilidad de educarse por el bien común no debe ser negocio propio del neoliberalismo. En ese sentido, Mejía et.al, (2017) en su estudio sobre los espacios de aprendizaje híbrido desarrollan nuevas metodologías que los docentes debieran poseer para desarrollar clases invertidas para el desarrollo de clases tecnológicas. Este planteamiento de educación nos invita a realizar un cambio donde los docentes no afrontan solos sino acompañados de una formación docente, seria y profunda para evitar la formación de educadores que no sean capaces de adaptarse a cambios generacionales tan profundos como los actuales.

En relación al contexto educativo, los profesores requieren de manera reglamentada, a nivel nacional e internacional, la implementación tecnológica y la formación para el uso de las tecnologías. A ello se agregan las condiciones propicias para ejercer el trabajo pedagógico fuera del aula virtual. Muchos 
docentes no conocen la producción de clases virtuales generando deficiencias en el sistema educativo. En el estudio de Belavi, et.al, (2020) se plantea que la falta de reconocimiento también se manifiesta en los distintos niveles de la educación, los profesores deben ser reconocidos por su labor e importancia en la educación.

El sistema educativo chileno está diseñado y ha sido ejecutado de forma presencial, en esta situación actual producto de la pandemia requiere de cambios metodológicos en la aplicación de la educación a distancia y la nueva implementación tanto de internet como de aparatos tecnológicos para el uso adecuado de la instrucción en línea, y la efectividad para el éxito pedagógico. Los establecimientos educacionales chilenos enseñan contenidos, se tratan dificultades de pobreza entregando alimentos, las instituciones pedagógicas brindan planes de salud Bucal u horas de atención para salud mental. Los establecimientos vulnerables de nuestro país cumplen más funciones que sólo enseñar y este diseño de atención múltiple hace perder el foco educativo. Debido a esto la relación interpersonal que se puede generar con este tipo de comunicaciones en vulnerabilidad puede ser peligrosa, la comunicación debe ser efectiva respecto de lo que se quiere lograr en cuanto al aprendizaje o a la asistencia social. Por su parte, Pinto Santos, et.al, (2017) han diseñado diferentes políticas públicas para la inclusión de los currículos escolares, donde la importancia está enfocada en la formación de los profesores.

Hoy la educación puede estar omnipresente con un profesor online u offline, plataformas virtuales, por cuanto los educandos manejan mucha información y necesitan aprender a distinguir la referencia fidedigna para anclarla a sus conocimientos. En esta etapa el docente debe manejar las tecnologías para situarse en la misma frecuencia tecnológica que los estudiantes. No se debe caer en los errores del pasado como fue hacer escribir con la pluma a los estudiantes cuando ya se había inventado el lápiz de mina. En resumen, en palabras de Abeysekera et.al, (2015) la educación debe ser activa para la efectividad del aprendizaje.

\section{Clases Virtuales, Didácticas innovadoras, evolución pedagógica}

La desigualdad en Chile, conocida principalmente a través de los medios de comunicación por ejemplo la radio Bio-Bio, comunicó el 27 de mayo del 2020 la noticia titulada como "El drama de alumnos que estudian en techos de casas para lograr señal de internet" donde comenta el problema de conexión que tienen muchos jóvenes para lograr conectarse a las clases virtuales, algunos de ellos, por ejemplo, deben subir al techo de sus casas para tener mejor conexión por la carente señal de internet que existen en zonas rurales de Chile. Los educandos deben subir al techo de sus casas incluso con frazadas y paraguas para no perder sus clases online, como país actualmente existe un problema de cobertura. Este problema no es desconocido para los docentes que deben entender este tipo de hechos y educarse para lograr el aprendizaje en los estudiantes que no están presentes en clases sincrónicas, para ello es necesario que la formación docente se fortalezca y los profesores logren obtener metodologías adecuadas para la instrucción de sus estudiantes. 
En la actualidad el acceso a las plataformas de comunicación son las aplicaciones de acceso cotidiano por ejemplo Facebook, mensajería instantánea, Twitter, estudiantes con teléfonos prepagos que acceden de manera más fácil a este tipo de aplicaciones cuando no tienen planes de internet contratados para conectarse a clases online. Los docentes que trabajan en zonas vulnerables deben incorporar a sus estudiantes en sus redes sociales digitales personales para ayudarlos en el aprendizaje de los educandos; ello se debe a que hoy existe una flexibilización en los medios de comunicación que se establecen entre estudiantes, colegas, y apoderados. De igual modo Serholt, et.al, (2017) relevan la experiencia de los docentes en Europa, y la aplicación de los robots en el ámbito educativo, hoy ya la tecnología esta instaurada en el aula, en el dialogo pedagógico, y en la comunicación para la ejecución del aprendizaje.

Para cambiar la forma de educar se requiere de una reformación docente, de nuevas investigaciones, metodologías de aprendizaje a distancia, adecuación de los tiempos de conexión, atención a la pantalla según la edad del educando. Seguir con el mismo modelo de la educación presencial con la única diferencia de ser transmitido por una pantalla virtual a distancia, no sería innovador. Por el contrario, los tiempos de concentración para los estudiantes podrían resultar perjudicial según lo publicado por la Academia Americana de Pediatría (AAP) en el 2018, la cual recomendó que los niños y jóvenes entre 6 y 18 años no pasaran más de 2 horas diarias frente a una pantalla. En consecuencia, es necesario repensar la entrega de contenidos sin tener que estar durante ocho horas diarias en clases online como se está haciendo actualmente en Chile para no generar problemas pediátricos en el futuro a causa del excesivo tiempo frente a las pantallas.

Junto a lo anterior, los profesores universitarios que imparten clases a los estudiantes de pedagogía deben actualizar sus conocimientos en plataformas virtuales, didácticas online, metodologías de aprendizaje a distancia para que los errores no sean en espiral. Bajo la perspectiva de una comunicación efectiva virtual se requiere de los docentes el manejo de competencias profesionales actualizadas. De igual manera, Revelo-Sánchez et.al, (2018) plantean que las TICs y la educación no pueden prescindir de la reflexión pedagógica, la innovación al aprendizaje colaborativo, puede ser aplicado en la educación online actual.

Lo que se observa en la actualidad es una evolución en la pedagogía, los docentes no pueden cometer errores en las plataformas online, porque las clases en muchas ocasiones quedan grabadas y los estudiantes aprenden erróneamente de forma sincrónica y asincrónica, deben ser capaces de lograr aprendizajes efectivos y de calidad en sus estudiantes. Los profesores que han debido vivir estar coyuntura histórica del cambio y revolución tecnológica con herramientas de aprendizaje, clases online y los estudiantes de pedagogía deben formarse en esta línea de conocimientos actuales con nuevas metodologías en la entrega de contenidos. Avello et.al, (2014) reconocen la importancia de la formación docente en las tecnologías como un sistema cíclico de avance clave para lograr las reformas educativas donde los profesores deben adquirir técnicas virtuales y sociales para el trabajo en red. 


\section{Plataformas educativas, virtualidad y efectividad}

La mayoría de los países han optado por la continuidad del proceso educativo mediante recursos en línea, el uso de Internet ofrece una oportunidad única y la cantidad de recursos pedagógicos que se pueden transformar en conocimiento, así como las diferentes herramientas de comunicación que proveen plataformas privilegiadas para acercar la escuela y los procesos educativos a los hogares y a los estudiantes en condiciones de confinamiento. A este respecto, UNESCO (2016) divulgó y promovió el uso del Marco de Competencias y Estándares TICs desde la Dimensión Pedagógica, otorgando importancia a la reflexión pedagógica.

Otro aspecto importante es el excesivo acercamiento virtual a lugares personales que deben ser abordados de forma ética y profesional en la comunicación. En este sentido, Briones Caicedo, et. al, (2020), plantean que el asertivo comunicacional va de la mano con la responsabilidad social, y la migración a la educación virtual donde los profesores alcancen a transmitir la efectividad, de modo que se consiga la comunicación eficaz.

Ahora es cuando los docentes que no adquirieron las habilidades comunicativas online puedan adquirirlas en el entendido que las habilidades y los aprendizajes pueden ser adquiridos sin mayor resistencia por parte del profesorado. Para establecer comunicación con los estudiantes y profesores pares, deben existir horarios y plataformas educativas establecidas, independiente del medio de comunicación ejercido, en caso de optar por mensajería instantánea o llamados telefónicos estos deben ser institucionales. Olcott Jr. et.al, (2015) hablan de la carretera cibernética, lo virtual, la comunicación educativa, que no suponen falsedad, ilusión o ficción, sino una nueva forma de ser que favorece procesos de creación más allá de lo que permite la presencialidad física.

La cultura multimedia y las múltiples plataformas que los jóvenes manejan de acuerdo a De Luca (2020) son espacios sociales actuales y la reflexión que debe tener el docente sobre los desafíos pedagógicos y determinan el futuro de la educación, pero también la sobrecarga actual de la tele-formación para abordar estos problemas preexistentes de la educación y las habilidades que deben poseer los educadores.

La instrucción telemática es una realidad actual, hoy es una pandemia, mañana podría ser una guerra, un desastre climático, terremotos u diferente tipo de cataclismo, por lo que es necesario sin duda percibir este problema actual de la pandemia como una oportunidad futura de progreso educativo pese a cualquier adversidad. La implementación online debe ser sin discriminación alguna, pues muchos estudiantes que viven en zonas extremas, y tienen dificultades de desplazamiento, la educación virtual mejora esas brechas de distancia. En este sentido autores como Torres-Ortiz, et.al, (2016) sostienen que los profesores deben adquirir herramientas pedagógicas para brindar clases a distancia, independiente del problema que se presente, el profesorado debe estar preparado para el futuro. 


\section{Rol docente, capacitación constante}

Los estudiantes actuales son nativos digitales, Millennials, Z y Alpha, y manejan tales aplicaciones, a diferencia de los docentes que están enfrentados a tecnologías con las cuales no crecieron, se trata de generaciones pasadas tales como la " $X$ " e " $Y$ ". Este problema generacional sobre el manejo de las tecnologías educativas, puede ser solucionado por lineamientos metodológicos para los docentes, especificando las habilidades que deben manejar los profesores para entender los códigos actuales de la comunicación virtual con sus estudiantes y de esta forma acercar las plataformas virtuales y los medios de comunicación. Ello permitirá que la tecnología sea una herramienta factible para establecer comunicación efectiva con los estudiantes y así brindar educación de calidad a distancia como un medio de transferencia de conocimiento. Las plataformas educativas deben establecer los medios educativos formales para que la vida personal no sea vulnerada por los medios de comunicación que Peters et.al, (1983) define como "comunicación masiva".

Además de lo anterior, la educación debe tener un enfoque dirigido hacia la enseñanza de las competencias actuales y futuras, la tecnológica estaba en marcha tiempo antes, pero la coyuntura de la pandemia aceleró los procesos educativos virtuales. Los profesores debemos manejar una comunicación efectiva respecto de nuestro trabajo, dejar de ofrecer resistencia a los cambios, bajo la premisa que "siempre el pasado fue mejor". De acuerdo con Almazán Gómez et.al, (2020) la digitalización alcanzó a todo el mundo, agilizando el proceso de digitalización pedagógica y Delors se adelantó en 1996 y relacionó la educación del siglo XXI, aprendizajes hoy ejecutados a través de la red de internet que toman sentido con los cuatro pilares de la educación fundamentales para la formación en línea.

Por su parte, Torres-Ortiz et.al, (2016) plantean que los contextos educativos son diversos con particularidades propias de las mediaciones tecnológicas y comunicacionales, y asocian las pedagogías instruccionales, constructivistas y humanistas como parte de los lineamientos propios de la calidad educativa. En este contexto los profesores deben ser capaces de transmitir conocimientos concentrados en menos tiempo y más eficaces, un ejemplo lo constituyen los videos llamativos que logren la atención del alumnado. Las clases expositivas virtuales deben evolucionar hacia nuevas experiencias cognitivas utilizando los recursos online para realizar clases online de calidad. Esta situación dependerá de la capacitación constante docente, para que la educación transite hacia una comunicación efectiva virtual.

De acuerdo con la literatura revisada, la formación del profesorado debe ser un tema central, pues en la actualidad quedó de manifiesto que debe modificarse para no caer en problemas de comunicación. La tecnología acerca distancias entre profesores y alumnos los cuales deben hablar el mismo lenguaje, y encontrarse en la sociedad de la información para avanzar como país y como región Latinoamérica. A este respecto, Suárez Huz et.al, (2018) conciben la actualización de los docentes y los estudiantes en función de los nuevos roles que desempeñan dicho enfoque educativo, las TICs son protagonistas de este espacio necesario para la formación constante docente. 


\section{CONCLUSIONES}

Es preciso avizorar una nueva realidad donde el uso de las tecnologías y las plataformas digitales llegaron para quedarse y el uso de estas herramientas será fundamental para la evolución y el éxito académico del país chileno. Por ello las clases online deberían estar enfocadas con sentido pedagógico para que los educandos puedan aprender y desarrollar la cognición frente a las pantallas, y los materiales pedagógicos deben ser fundamentales para el profesorado. En 1922, Tomás A. Edison predijo que el cine remplazaría los libros y tal vez incluso a los maestros, un profesor podría hacer el trabajo de cien, si la tecnología es bien utilizada.

Un gran reto para la consolidación docente no es adaptar sus clases en salas de clases presenciales a las plataformas online; además, el llamado es a cambiar e innovar la forma para conseguir el aprendizaje con mayor efectividad, calidad educativa y mejorando los tiempos de aprendizaje. La educación virtual requiere de tiempos de planificación y producción de material educativo para clases online y offline, y apoyado por una formación docente constante porque la tecnología avanza rápidamente y las necesidades pedagógicas son infinitas.

\section{REFERENCIAS}

Abeysekera, L., \& Dawson, P. (2015). Motivation and cognitive load in the flipped classroom: definition, rationale and a call for research. Higher education research \& development, 34(1), 1-14.

Almazán, A. (2020). Covid-19: ¿Punto Sin Retorno de la Digitalización de la Educación?. Revista Internacional De Educación Para La Justicia Social, 9(3).

Avello, R, López, R, Álvarez, H, Vázquez, S, Gómez, A, \& Alpízar, R. (2014). Experiencia cubana sobre la formación del docente latinoamericano en tecnologías para la educación. Educación Médica Superior, 28(3), 587-591.

Belavi, G., \& Murillo, F. J. (2020). Democracia y Justicia Social en las Escuelas: Dimensiones para Pensar y Mejorar la Práctica Educativa. REICE. Revista Iberoamericana Sobre Calidad, Eficacia Y Cambio En Educación, 18(3), 5-28.

Carrera, J. (2020), "Con paraguas": El drama de alumnos estudian en techos de casas para lograr señal de internet, Biobiochile.cl, La Radio. Chile.

Briones, W., Urquiza, L., Navia, J., Huilcapi, M., Muñoz, G., \& Zambrano, R. (2020). Gestión de la comunicación asertiva en la labor extensionista en las instituciones de educación superior. Journal of Science and Research: Revista Ciencia E Investigación. ISSN 2528-8083, 5(2), 1 - 16.

Del Prado, L. (2017). CICS/IED/UNESCO (2016). Informe Mundial sobre Ciencias Sociales - Afrontar el reto de las desigualdades y trazar vías hacia un mundo justo. París: UNESCO (Francia). AZARBE, Revista Internacional de Trabajo Social y Bienestar, (6), 99-100.

Delors, J. (1996). Los cuatro pilares de la educación. En Delors et al. La educación encierra un tesoro. Informe a la UNESCO de la Comisión internacional sobre la educación para el siglo XXI, (pp. 91103). Madrid: Santillana/UNESCO.

De Luca, M. (2020). Las aulas virtuales en la formación docente como estrategia de continuidad pedagógica en tiempos de pandemia. Usos y paradojas. Análisis Carolina, 2020(33), 1-12. 
Gudgel, Dan (2018), Nuevas recomendaciones para el consumo mediático de los niños, American Academy of Pediatrics. https://www.aao.org/salud-ocular/consejos/uso-de-pantalla-para-los-ninos

Hurtado, F. (2020). La educación en tiempos de pandemia: los desafíos de la escuela del siglo XXI. Revista Arbitrada del Centro de Investigación y Estudios Gerenciales (44). Obtenido de http://www. grupocieg.org/archivos_revista/Ed.44(176-187)

Mejía, C., Michalón, D., Michalón, R., López, R., Palmero, D., \& Sánchez, S. (2017). Espacios de aprendizaje híbridos. Hacia una educación del futuro en la Universidad de Guayaquil. Medisur, 15(3), 350-355.

Munro, M. (2018). The complicity of digital technologies in the marketisation of UK higher education: exploring the implications of a critical discourse analysis of thirteen national digital teaching and learning strategies. International Journal of Educational Technology in Higher Education, 15, 1-20.

OECD (2020), TALIS 2018 Results (Volume II): Teachers and School Leaders as Valued Professionals, TALIS, OECD Publishing, Paris, https://doi.org/10.1787/19cf08df-en.

Olcott Jr. D., Carrera Farran, X., Gallardo Echenique, E. E. y González Martínez, J. (2015). Ética y Educación en la era digital: perspectivas globales y estrategias para la transformación local en Cataluña. RUSC. Universities and Knowledge Society Journal, 12(2). págs. 59-72.

Peters, R. (1983) R. S.,Philosophy of Education, en Hirst, P. (ed.), Educational Theory and its Foundation Disciplines, (pp. 30-61). London: Routledge \& Kegan Paul.

Pinto, A., Cortés, O., \& Alfaro, C. (2017). Hacia la transformación de la práctica docente: modelo espiral de competencias TICTACTEP. Pixel-Bit. Revista De Medios Y Educación, (51), 37-51.

Reimers, F. y Schleider, A. (2020). Un marco para guiar una respuesta educativa a la pandemia del 2020 del COVID-19. OECD. https://www.oei.es/Ciencia/Noticia/la-oei-difunde-un-informede-launiversidad-de-harvard

Revelo,O,Collazos, C.,\&Jiménez,J.(2018).Eltrabajocolaborativocomoestrategiadidáctica paralaenseñanza/ aprendizaje de la programación: una revisión sistemática de literatura. TecnoLógicas, 21(41), 115 134.

Serholt, S; Barendregt, W; Vasalou, A; Alves, P; Jones, A; Petisca, S; Paiva, A; (2017) The case of classroom robots: teachers' deliberations on the ethical tensions. Al and Society , 32 (4) pp. 613-631.

Suárez, Y. (2018). Impacto de la Educación Virtual y las TIC en la Andragogía. Revista Ciencias de la Educación, 28(51), 326-347.

Torres, J., \& Duarte, J. (2016). Los procesos pedagógicos administrativos y los aspectos socio-culturales de inclusión y tecno-pedagogía a través de las tendencias pedagógicas en educación a distancia y virtual. Revista de investigación, desarrollo e innovación, 6(2), 179-190.

Villarreal, S, García, J, Hernández, H, \& Steffens, E. (2019). Competencias Docentes y Transformaciones en la Educación en la Era Digital. Formación universitaria, 12(6), 3-14. 